\title{
Direct and converse results in the $B a$ space for Jackson-Matsuoka polynomials on the unit sphere
}

Guo Feng $^{1 *}$ and Yuan Feng ${ }^{2}$

\section{"Correspondence:} gfeng@tzc.edu.cn

${ }^{1}$ Department of Mathematics,

Taizhou University, Taizhou,

Zhejiang 317000, China

Full list of author information is

available at the end of the article

\begin{abstract}
In this paper, we introduce $K$-functional and modulus of smoothness of the unit sphere in the $B a$ space, establish their relations and obtain the direct and converse theorem of approximation in the Ba space for Jackson-Matsuoka polynomials on the unit sphere of $\mathbb{R}^{d}$
\end{abstract}

MSC: $41 \mathrm{~A} 25 ; 41 \mathrm{~A} 35 ; 41 \mathrm{~A} 63$

Keywords: Jackson-Matsuoka polynomials; Ba space; modulus of smoothness; $K$-functional; spherical means

\section{Introduction}

Let $\mathbb{S}:=\mathbb{S}^{d-1}=\{x:\|x\|=1\}$ denote the unit sphere in $\mathbb{R}^{d}(d \geq 3), d \in \mathbb{N}$, where $\|x\|$ denotes the usual Euclidean norm, $\mathbb{Z}_{+}$the set of nonnegative integers, and $\mathbb{N}$ the set of positive integers. We denote by $L_{p}:=L_{p}(\mathbb{S}), 1 \leq p \leq \infty$, the space of functions defined on $\mathbb{S}$ with the finite norm

$$
\|f\|_{p}:= \begin{cases}\left(\int_{\mathbb{S}}|f(\varpi)|^{p} d \varpi\right)^{\frac{1}{p}}, & 1 \leq p<\infty, \\ {\operatorname{ess} \sup _{\varpi \in \mathbb{S}}|f(\varpi)|,} \quad p=\infty,\end{cases}
$$

where $\varpi \in \mathbb{S}$, and $d \varpi$ is the measure element on $\mathbb{S}$, and $\left|\mathbb{S}^{d-1}\right|=\int_{\mathbb{S}} d \varpi=\frac{2 \pi^{\frac{d}{2}}}{\Gamma\left(\frac{d}{2}\right)}$ is the surface area of $\mathbb{S}$.

The conception of $B a$ space was first put forward by Ding and Luo (see [1]) in their discussion of the prior estimate of Laplace operator in some classical domains and in their study of the embedding theorem of Orlicz-Sobolev spaces, higher dimensional singular integrals, and harmonic function etc.

Definition 1.1 (see [1]) Let $B=\left\{B_{1}, B_{2}, \ldots, B_{m}, \ldots\right\}$ be a sequence of linear normed function spaces, $a=\left\{a_{1}, a_{2}, \ldots, a_{m}, \ldots\right\}$ be a sequence of nonnegative numbers. For $f \in$ $\bigcap_{m=1}^{\infty} B_{m}$, we form the power series of

$$
I(f, \alpha):=\sum_{m=1}^{\infty} a_{m} \alpha^{m}\|f\|_{B_{m}}^{m} .
$$

If $I f(\alpha)$ has a non-zero radius of convergence, we say $f \in B a$.

\section{Springer}

(c) 2014 Feng and Feng; licensee Springer. This is an Open Access article distributed under the terms of the Creative Commons Attribution License (http://creativecommons.org/licenses/by/2.0), which permits unrestricted use, distribution, and reproduction in any medium, provided the original work is properly cited. 
The norm in $B a$ is defined by

$$
\|f\|_{B a}:=\inf _{\alpha>0}\left\{\frac{1}{\alpha}: I(f, \alpha) \leq 1\right\}
$$

As proved in [1], $B a$ is a Banach space if $B_{m}$ is a Banach space. Evidently, if $B_{m}=L_{m}$, then $B a$ space is an Orlicz space. If $B_{m}=L_{p}, a=\{1,0, \ldots, 0, \ldots\}$, then a $B a$ space is a classical Lebesgue space.

Hereafter the space of spherical harmonics of degree $k$ is denoted by $\mathcal{H}_{k}^{d}$. The LaplaceBeltrami operator on the unit sphere is denoted by

$$
\mathfrak{D} f(\varpi):=\left.\Delta f\left(\frac{\varpi}{|\varpi|}\right)\right|_{\varpi \in \mathbb{S}},
$$

which has eigenvalue $\lambda_{k}:=-k(k+d-2)$ corresponding to the eigenspace $\mathcal{H}_{k}^{d}$ with $k \in$ $\mathbb{Z}_{+}$, namely, $\mathcal{H}_{k}^{d}=\{\Psi \in C(\mathbb{S}): \mathfrak{D} \Psi=-k(k+d-2) \Psi\}$. For the properties of the space of spherical harmonics and the Laplace-Beltrami operators, see [2-4]. The standard Hilbert space theory shows that $L_{2}(\mathbb{S})=\sum_{k=0}^{\infty} \bigoplus \mathcal{H}_{k}^{d}$. The orthogonal projection $Y_{k}: L_{2}(\mathbb{S}) \mapsto \mathcal{H}_{k}^{d}$ takes the form

$$
Y_{k}(f ; \varpi):=\frac{\Gamma(\lambda)(k+\lambda)}{2 \pi^{\lambda+1}} \int_{\mathbb{S}} P_{k}^{\lambda}(\varpi, \vartheta) f(\vartheta) d \vartheta,
$$

where $2 \lambda=d-2, P_{k}^{\lambda}$ denotes hyperspherical polynomials of degree $k$ which satisfies $(1-$ $\left.2 r \cos \theta+r^{2}\right)^{-r}=\sum_{k=0}^{\infty} r^{k} P_{k}^{\lambda}(\cos \theta), 0 \leq \theta \leq \pi$.

The spherical means are denoted by

$$
T_{\theta}(f):=T_{\theta}(f ; \varpi):=\frac{1}{\left|\mathbb{S}^{d-2}\right|(\sin \theta)^{d-2}} \int_{\langle\varpi, \vartheta\rangle=\cos \theta} f(\vartheta) d \vartheta
$$

where $\left|\mathbb{S}^{d-2}\right|$ is the surface area of $\mathbb{S}^{d-2},\langle x, y\rangle$ denotes the usual Euclidean inner product. The properties of the spherical means are well known (see $[5,6])$.

Based on the classical Jackson-Matsuoka kernel (see [7]) we define a new kernel

$$
M_{n ; j, i, s}(\theta):=\frac{1}{\Omega_{n ; j, i, s}}\left(\frac{\sin ^{2 j} n \theta / 2}{\sin ^{2 i} \theta / 2}\right)^{2 s}, \quad n=1,2, \ldots, \theta \in \mathbb{R}
$$

where $j, i, s \in \mathbb{N}, \Omega_{n ; j, i, s}$ is chosen such that $\int_{0}^{\pi} M_{n ; j, i, s}(\theta) \sin ^{2 \lambda} \theta d \theta=1$. It is well known that $M_{n ; j, i, s}(\theta)$ is an even nonnegative operator. In particular, it is an even and nonnegative trigonometric polynomial of degree at most $2 s(n j+2 j-2 i)$ for $j \geq i$ and the Jackson polynomial for $j=i$. Using $M_{n ; j, i, s}(\theta)$ we consider spherical convolution:

$$
J_{n ; j, i, s}(f ; \varpi):=\left(f * M_{n ; j, i, s}\right)(\varpi):=\int_{0}^{\pi} T_{\theta}(f ; \varpi) M_{n ; j, i, s}(\theta)(\theta) \sin ^{2 \lambda} \theta d \theta
$$

It is called the Jackson-Matsuoka polynomial on the unit sphere based on the JacksonMatsuoka kernel. In particular, $\left(f_{0} * M_{n ; j, i, s}\right)(\varpi)=1$ for $f_{0}(\varpi)=1$. The classical JacksonMatsuoka polynomial in classical $L_{p}$ space has been studied by many authors (see $\left.[7,8]\right)$. In this paper, we consider the approximation of the Jackson-Matsuoka polynomial on the unit sphere in the $B a$ space. Firstly, we introduce $K$-functionals, modulus of smooth- 
ness on the unit sphere in the $B a$ space, establish their relations. Then with the help of the relation between $K$-functionals and modulus of smoothness on the sphere in the $B a$ space and the properties of the spherical means, we obtain the direct and converse best approximation in the $B a$ space by Jackson-Matsuoka polynomial on the unit sphere of $\mathbb{R}^{d}$.

\section{$2 K$-Functionals and modulus of smoothness}

Definition 2.1 For $f \in B a$, the modulus of smoothness on the unit sphere is given by

$$
\omega(f ; t)_{B a}:=\sup _{0<\theta \leq t}\left\|f-T_{\theta}(f)\right\|_{B a} .
$$

The $K$-functional of the unit sphere is given by

$$
K\left(f ; t^{2}\right)_{B a}:=\inf _{g \in W_{B a}(\mathbb{S})}\left\{\|f-g\|_{B a}+t^{2}\|\mathfrak{D} g\|_{B a}\right\},
$$

where $W_{B a}(\mathbb{S}):=\{f: f \in B a, \mathfrak{D} f \in B a\}, 0<t<t_{0}, t_{0}$ is a positive constant, $\mathfrak{D} f$ denotes the Laplace-Beltrami operator on the unit sphere.

To prove the weak equivalence between the $K$-functional and the modulus of smoothness on the unit sphere, we need the following lemma.

Lemma 2.2 Let $B=\left\{L_{p_{1}}, L_{p_{2}}, \ldots, L_{p_{m}}, \ldots\right\}$ be a sequence of Lebesgue spaces, $p_{m} \geq 1, m=$ $1,2, \ldots, a=\left\{a_{1}, a_{2}, \ldots, a_{m}, \ldots\right\}$ be a sequence of nonnegative numbers, $\left\{a_{m}^{\frac{1}{m}}\right\} \in l^{\infty},\left\{a_{m}^{-\frac{1}{m}}\right\} \in$ $l^{\infty}$. Iff $\in B a:=\bigcap_{m=1}^{\infty} L_{p_{m}}$, then

$$
\|f\|_{p_{m}} \leq \frac{1}{\mu}\|f\|_{B a},
$$

where $\mu=\inf _{m \geq 1}\left\{a_{m}^{\frac{1}{m}}\right\}$.

Proof Since $\left\{a_{m}^{\frac{1}{m}}\right\} \in l^{\infty}$, we may let $0<q=\sup _{m \geq 1}\left\{a_{m}^{\frac{1}{m}}\right\} \in L^{\infty}$. From $\left\{a_{m}^{-\frac{1}{m}}\right\} \in l^{\infty}$, we may let $\mu=\inf _{m \geq 1}\left\{a_{m}^{\frac{1}{m}}\right\}$. Then $0<\mu<\infty$.

In view of the $\sum_{m=1}^{\infty} a_{m} \alpha^{m}\|f\|_{p_{m}}^{m} \leq 1$, the $\sup _{m \geq 1}\|f\|_{p_{m}}$ exists. Let

$$
u=\sup _{m \geq 1}\left\{\|f\|_{p_{m}}\right\} .
$$

By the definition of supremum, for any $\delta>0$, there exists $K \geq 1$, such that $\|f\|_{p_{K}}>u-\delta$. By the definition of $\|f\|_{B a}=\inf _{\alpha>0}\left\{\frac{1}{\alpha}: I(f, \alpha) \leq 1\right\}$, for any $\varepsilon>0$, there exists $\frac{1}{\alpha_{1}}$, such that $\sum_{m=1}^{\infty} a_{m} \alpha_{1}^{m}\|f\|_{p_{m}}^{m} \leq 1$ holds. Therefore $\|f\|_{B a}=\inf _{\alpha>0}\left\{\frac{1}{\alpha}: I(f, \alpha)\right\}>\frac{1}{\alpha_{1}}-\varepsilon$. Namely

$$
1 \geq \sum_{m=1}^{\infty} a_{m} \alpha_{1}^{m}\|f\|_{p_{m}}^{m} \geq a_{K} \alpha_{1}^{K}\|f\|_{p_{K}}^{K}>\left[a_{K}^{\frac{1}{K}}(u-\delta)\right]^{K} \geq\left[s \alpha_{1}(u-\delta)\right]^{K}
$$

By the arbitrariness of $\delta$,

$$
\begin{aligned}
& \frac{1}{\alpha_{1}} \geq \mu \cdot u=\mu \cdot \sup _{m \geq 1}\left\{\|f\|_{p_{m}}\right\}, \\
& \|f\|_{p_{m}}>\frac{1}{\alpha_{1}}-\varepsilon \geq \mu \cdot \sup _{m \geq 1}\left\{\|f\|_{p_{m}}\right\}-\varepsilon,
\end{aligned}
$$


and also $\varepsilon$ is arbitrary, therefore

$$
\sup _{m \geq 1}\left\{\|f\|_{p_{m}}\right\} \leq \frac{1}{\mu}\|f\|_{B a},
$$

which implies that for any $p_{m}$, we have

$$
\|f\|_{p_{m}} \leq \frac{1}{\mu}\|f\|_{B a} .
$$

The proof is completed.

We will establish the weak equivalence between the $K$-functional and the modulus of smoothness on the unit sphere in the $B a$ space.

Theorem 2.3 Let $B=\left\{L_{p_{1}}, L_{p_{2}}, \ldots, L_{p_{m}}, \ldots\right\}$ be a sequence of Lebesgue spaces, $p_{m} \geq 1, m=$ $1,2, \ldots, a=\left\{a_{1}, a_{2}, \ldots, a_{m}, \ldots\right\}$ be a sequence of nonnegative numbers. If $\left\{a_{m}^{\frac{1}{m}}\right\} \in l^{\infty},\left\{a_{m}^{-\frac{1}{m}}\right\} \in$ $l^{\infty}$. Then for $f \in B a, 0<t<\frac{\pi}{2}$, the weak equivalence

$$
\omega(f ; t)_{B a} \asymp K\left(f ; t^{2}\right)_{B a}
$$

holds, where the weakly equivalent relation $A(n) \asymp B(n)$ means that $A(n) \ll B(n)$ and $B(n) \ll A(n)$, and relation $A_{n} \ll B_{n}$ means that there is a positive constant $C$ independent on $n$ such that $A(n) \leq C B(n)$ holds.

Throughout this paper, $C$ denotes a positive constant independent on $n$ and $f$ and $C(a)$ denotes a positive constant dependent on $a$, which may be different according to the circumstances.

Proof For $m=1,2, \ldots, g \in W_{B a}(\mathbb{S})$, note that [9]

$$
\begin{aligned}
& \left\|T_{\theta} g-g\right\|_{p_{m}} \leq C \theta^{2}\|\mathfrak{D} g\|_{p_{m}}, \\
& \left\|T_{\theta} f\right\|_{p_{m}} \leq\|f\|_{p_{m}} .
\end{aligned}
$$

By the definition of the $B a$-norm $\|\cdot\|_{B a}$ and (2.3), we have

$$
\begin{aligned}
\left\|T_{\theta} g-g\right\|_{B a} & =\inf _{\alpha>0}\left\{\alpha: \sum_{m=1}^{\infty} \frac{a_{m}}{\alpha^{m}}\left\|T_{\theta} g-g\right\|_{p_{m}}^{m} \leq 1\right\} \\
& \leq \inf _{\alpha>0}\left\{\alpha: \sum_{m=1}^{\infty} \frac{a_{m}}{\alpha^{m}} C^{m} \theta^{2 m}\|\mathfrak{D} g\|_{p_{m}}^{m} \leq 1\right\} \\
& \leq \inf _{\alpha>0}\left\{\alpha: \sum_{m=1}^{\infty} \frac{C^{m} q^{m}}{\alpha^{m}} \theta^{2 m}\|\mathfrak{D} g\|_{p_{m}}^{m} \leq 1\right\} \\
& \leq \inf _{\alpha>0}\left\{\alpha: \sum_{m=1}^{\infty} \frac{1}{\alpha^{m}}\left(\frac{C \cdot q \cdot \theta^{2}}{\mu}\|\mathfrak{D} g\|_{B a}\right)^{m} \leq 1\right\} .
\end{aligned}
$$


Let $\alpha=2 \frac{C \cdot q \cdot \theta^{2}}{\mu}\|\mathfrak{D} g\|_{B a}$, then $\sum_{m=1}^{\infty} \frac{1}{\alpha^{m}}\left(\frac{C \cdot q \cdot \theta^{2}}{\mu}\|\mathfrak{D} g\|_{B a}\right)^{m}=1$. Consequently $\sum_{m=1}^{\infty} \frac{a_{m}}{\alpha^{m}} \| T_{\theta} g-$ $g \|_{p_{m}}^{m} \leq 1$. Therefore, we have

$$
\left\|T_{\theta} g-g\right\|_{B a} \leq C(q, \mu) \theta^{2}\|\mathfrak{D} g\|_{B a} .
$$

The proof is similar to that of (2.6), we get

$$
\left\|T_{\theta}(f-g)\right\|_{B a} \leq C(q, \mu)\|f-g\|_{B a} .
$$

The triangle inequality gives

$$
\left\|T_{\theta} f-f\right\|_{B a} \leq 2\|f-g\|_{B a}+C(q, \mu) \theta^{2}\|\mathfrak{D} g\|_{B a},
$$

which shows that $\omega(f ; t)_{B a} \leq C(q, \mu) K\left(f ; t^{2}\right)_{B a}$. On the other hand, we define

$$
g(x)=v_{0} \int_{0}^{\theta}(\sin u)^{-2 \lambda} d u \int_{0}^{u} T_{t} f(x)(\sin t)^{2 \lambda} d t
$$

with $v_{\theta}^{-1}=\int_{0}^{\theta}(\sin u)^{-2 \lambda} d u \int_{0}^{u}(\sin t)^{2 \lambda} d t$. Then $\mathfrak{D} g=v_{\theta}\left(T_{\theta} f-f\right)$, this also gives

$$
\|\mathfrak{D} g\|_{p_{m}} \leq C \theta^{-2}\left\|T_{\theta} f-f\right\|_{p_{m}} .
$$

Since for $0 \leq \theta \leq \frac{\pi}{2}$, the inequality $\frac{2}{\pi} \theta \leq \sin \theta \leq \theta$ shows that $v_{\theta}^{-1} \asymp \theta^{2}$. Moreover,

$$
f-g=v_{\theta}^{-1} \int_{0}^{\theta}(\sin u)^{-2 \lambda} d u \int_{0}^{u}\left(T_{t}-f\right)(\sin t)^{2 \lambda} d t .
$$

Consequently, we get

$$
\|f-g\|_{p_{m}} \leq C\left\|T_{\theta} f-f\right\|_{p_{m}} .
$$

By (2.8) and (2.9), similar to the proof of (2.6), we obtain

$$
\|\mathfrak{D} g\|_{B a} \leq C \theta^{-2}\left\|T_{\theta} f-f\right\|_{B a}
$$

and

$$
\|f-g\|_{B a} \leq C\left\|T_{\theta} f-f\right\|_{B a} .
$$

Combining (2.10), (2.11), and the definition of $K$-functional, we have

$$
\begin{aligned}
K\left(f ; \theta^{2}\right)_{B a} & \leq\|f-g\|_{B a}+\theta^{2}\|\mathfrak{D} g\|_{B a} \\
& \leq C\left\|T_{\theta} f-f\right\|_{B a}+C \theta^{-2} \theta^{2}\left\|T_{\theta} f-f\right\|_{B a} \\
& \leq C\left\|T_{\theta} f-f\right\|_{B a} .
\end{aligned}
$$

Thus

$$
K\left(f ; t^{2}\right)_{B a} \leq C \omega(f ; t)_{B a} .
$$


Corollary 2.4 For $t \geq 0$, there is a constant $C$ such that

$$
\omega(f ; t \delta)_{B a} \leq C \max \left\{1, t^{2}\right\} \omega(f ; \delta)_{B a} .
$$

Proof By the weakly equivalent relation between the modulus of smoothness and $K$-functional, and the definition of $K\left(f ; t^{2}\right)_{B a}$, we have

$$
\begin{aligned}
\omega(f ; t \delta)_{B a} & \leq C K\left(f ;(t \delta)^{2}\right)_{B a} \leq C\left(\|f-g\|_{B a}+t^{2} \delta^{2}\|\mathfrak{D} g\|_{B a}\right) \\
& \leq C \max \left\{1, t^{2}\right\}\left(\|f-g\|_{B a}+\delta^{2}\|\mathfrak{D} g\|_{B a}\right) \\
& \leq C \max \left\{1, t^{2}\right\} K\left(f ; \delta^{2}\right)_{B a} \leq C \max \left\{1, t^{2}\right\} \omega(f ; \delta)_{B a} .
\end{aligned}
$$

Corollary 2.4 has been proved.

\section{Some lemmas}

Lemma 3.1 Let $\Omega_{n ; j, i, s}=\int_{0}^{\pi}\left(\frac{\sin ^{2 j} \frac{n \theta}{2}}{\sin ^{2 i} \frac{\theta}{2}}\right)^{2 s} \sin ^{2 \lambda} \theta d \theta$. Then the weak equivalence

$$
\Omega_{n ; j ; i, S} \asymp n^{4 i s-2 \lambda-1}
$$

holds for $4 s i>2 \lambda+1, j \geq i$.

Proof As $\frac{\theta}{\pi} \leq \sin \frac{\theta}{2} \leq \frac{\theta}{2}$, and $\sin \theta \leq \theta$ for $0 \leq \theta \leq \pi$, we have

$$
\begin{aligned}
\Omega_{n ; j, i, s} & =\int_{0}^{\pi}\left(\frac{\sin ^{2 j} \frac{n \theta}{2}}{\sin ^{2 i} \frac{\theta}{2}}\right)^{2 s} \sin ^{2 \lambda} \theta d \theta \\
& \asymp n^{4 i s-2 \lambda-1} \int_{0}^{n \pi / 2} t^{2 \lambda}\left(\frac{\sin ^{2 j} t}{t^{2 i}}\right)^{2 s} d t \\
& \asymp n^{4 i s-2 \lambda-1}\left(\int_{0}^{\pi / 2} t^{2 \lambda}\left(\frac{\sin ^{2 j} t}{t^{2 i}}\right)^{2 s} d t+\int_{\pi / 2}^{\infty} t^{2 \lambda}\left(\frac{\sin ^{2 j} t}{t^{2 i}}\right)^{2 s} d t\right) \\
& \asymp n^{4 i s-2 \lambda-1},
\end{aligned}
$$

since $4 s i>2 \lambda+1, j \geq i$. Lemma 3.1 has been proved.

Lemma 3.2 For $4 i s>r+2 \lambda+1, j \geq i, r \in \mathbb{R}$, there is a constant $C(\lambda, j, i, s)$ such that

$$
\int_{0}^{\pi} \theta^{r} M_{n ; j, i, s}(\theta) \sin ^{2 \lambda} \theta d \theta \leq C(\lambda, j, i, s) n^{-r} .
$$

Proof Since $\frac{\theta}{\pi} \leq \sin \frac{\theta}{2} \leq \frac{\theta}{2}$, and $\sin \theta \leq \theta$ for $0 \leq \theta \leq \pi$, by $\Omega_{n ; j, i, s} \asymp n^{4 i s-2 \lambda-1}$, we have

$$
\begin{aligned}
& \int_{0}^{\pi} \theta^{r} M_{n ; j ;, i s}(\theta) \sin ^{2 \lambda} \theta d \theta \\
& \quad \leq C(\lambda, i, j, s) n^{-4 i s+2 \lambda+1} \int_{0}^{\pi} \theta^{r}\left(\frac{\sin ^{2 j} \frac{n \theta}{2}}{\sin ^{2 i} \frac{\theta}{2}}\right)^{2 s} \sin ^{2 \lambda} \theta d \theta \\
& \leq C(\lambda, i, j, s) n^{-4 i s+2 \lambda+1} n^{4 i s-r-2 \lambda-1} \int_{0}^{n \pi / 2} t^{r+2 \lambda}\left(\frac{\sin ^{2 j} t}{t^{2 i}}\right)^{2 s} d t
\end{aligned}
$$




$$
\begin{aligned}
& \leq C(\lambda, i, j, s) n^{-r}\left(\int_{0}^{\pi / 2} t^{r+2 \lambda}\left(\frac{\sin ^{2 j} t}{t^{2 i}}\right)^{2 s} d t+\int_{\pi / 2}^{\infty} t^{r+2 \lambda}\left(\frac{\sin ^{2 j} t}{t^{2 i}}\right)^{2 s} d t\right) \\
& \leq C(\lambda, j, i, s) C_{2} n^{\lambda} \leq C(\lambda, j, i, s) n^{\lambda}
\end{aligned}
$$

where

$$
C_{2}=\int_{0}^{\pi / 2} t^{\lambda}\left(\frac{\sin ^{2 j} t}{t^{2 i}}\right)^{2 s} d t+\int_{\pi / 2}^{\infty} t^{\lambda}\left(\frac{\sin ^{2 j} t}{t^{2 i}}\right)^{2 s} d t, \quad 4 i s>r+2 \lambda+1, j \geq i .
$$

Lemma 3.3 (see [9]) Suppose that $g \in C^{2}(\mathbb{S})$. Then, for $\varpi \in(S)$ and $0<t<\frac{\pi}{2}$, we have

$$
\begin{aligned}
& B_{t}(g, \varpi)-g(\varpi)=\frac{1}{\Phi(t)} \int_{0}^{t} \sin ^{d-2} \theta d \theta \int_{0}^{\theta} \frac{1}{\sin ^{d-2} u} \Phi(u) B_{u}(\mathfrak{D} g, \varpi) d u, \\
& T_{\theta}(g ; \varpi)-g(\varpi)=\frac{\Gamma\left(\frac{d-1}{2}\right)}{2 \pi^{\frac{d-1}{2}}} \int_{0}^{\theta} \frac{\Phi(t)}{\sin ^{d-2} t} B_{t}(\mathfrak{D} g, \varpi) d t
\end{aligned}
$$

where

$$
\begin{aligned}
& B_{t}(f, \varpi)=\frac{1}{\Phi(t)} \int_{\cos t \leq\langle\varpi, \vartheta\rangle \leq 1} f(\vartheta) d \vartheta, \quad t>0, \varpi, \vartheta \in \mathbb{S}^{d-1}, \\
& \Phi(t)=\frac{2 \pi^{\frac{d-1}{2}}}{\Gamma\left(\frac{d-1}{2}\right)} \int_{0}^{t} \sin ^{d-2} u d u .
\end{aligned}
$$

Lemma 3.4 Let $g, \mathfrak{D} g, \mathfrak{D}^{2} g \in B a, B a:=\bigcap_{m=1}^{\infty} L_{p_{m}}(\mathbb{S}), m=1,2, \ldots, 1 \leq p_{m} \leq \infty, J_{n ; j, i, s}(f ; \varpi)$ be the Jackson-Matsuoka polynomial on the unit sphere based on the Jackson-Matsuoka kernel, $4 i s>d+3$. Then there is a constant $C(d, j, i, s)$ such that

$$
\left\|J_{n ; j, i, s} g-g-\alpha(n) \mathfrak{D} g\right\|_{B a} \leq C(d, j, i, s) n^{-4}\left\|\mathfrak{D}^{2} g\right\|_{B a},
$$

where $\alpha(n) \asymp n^{-2}$.

Proof For $m \in \mathbb{N}$, by (3.5), we have

$$
\begin{aligned}
& J_{n ; j, i, s}(g ; \varpi)-g(\varpi) \\
&=\int_{0}^{\pi} M_{n ; j, i, s}(\theta)\left(T_{\theta}(g ; \varpi)-g(\varpi)\right) \sin ^{d-2} \theta d \theta \\
&=\int_{0}^{\pi} M_{n ; j, i, s}(\theta) \sin ^{d-2} \theta d \theta \frac{\Gamma\left(\frac{d-1}{2}\right)}{2 \pi \frac{d-1}{2}} \int_{0}^{\theta} \frac{\Phi(t)}{\sin ^{d-2} t} B_{t}(\mathfrak{D} g, \varpi) d t \\
&=\operatorname{Dg} g(\varpi) \int_{0}^{\pi} M_{n ; j, i, s}(\theta) \sin ^{d-2} \theta d \theta \frac{\Gamma\left(\frac{d-1}{2}\right)}{2 \pi^{\frac{d-1}{2}}} \int_{0}^{\theta} \frac{\Phi(t)}{\sin ^{d-2} t} d t \\
& \quad+\int_{0}^{\pi} M_{n ; j, i, s}(\theta) \sin ^{d-2} \theta d \theta \frac{\Gamma\left(\frac{d-1}{2}\right)}{2 \pi \frac{d-1}{2}} \int_{0}^{\theta} \frac{\sin ^{d-2} t}{t}\left(B_{t}(\mathfrak{D} g, \varpi)-\mathfrak{D} g(\varpi)\right) d t \\
& \quad \times \int_{0}^{t} \sin ^{d-2} u\left(B_{t}(\mathfrak{D} g, \varpi)-\mathfrak{D} g(\varpi)\right) d u \\
&= \mathfrak{D} g(\varpi) \int_{0}^{\pi} M_{n ; j, i, s}(\theta) \sin ^{d-2} \theta d \theta \int_{0}^{\theta} \frac{d t}{\sin ^{d-2} t} \int_{0}^{t} \sin ^{d-2} u d u
\end{aligned}
$$




$$
\begin{aligned}
& +\int_{0}^{\pi} M_{n ; j, i, s}(\theta) \sin ^{d-2} \theta d \theta \int_{0}^{\theta} \frac{d t}{\sin ^{d-2} t} \int_{0}^{t} \sin ^{d-2} u\left(B_{t}(\mathfrak{D} g, \varpi)-\mathfrak{D} g(\varpi)\right) d u \\
:= & \alpha(n) \mathfrak{D} g(\varpi)+\int_{0}^{\pi} M_{n ; j, i, s}(\theta) \sin ^{d-2} \theta \Psi_{\theta}(g, \varpi) d \theta
\end{aligned}
$$

where

$$
\alpha(n):=\int_{0}^{\pi} M_{n ; j, i, s}(\theta) \sin ^{d-2} \theta d \theta \int_{0}^{\theta} \frac{d t}{\sin ^{d-2} t} \int_{0}^{t} \sin ^{d-2} u d u
$$

and

$$
\begin{aligned}
& \Psi_{\theta}(g, \varpi):=\int_{0}^{\theta} \frac{d t}{\sin ^{d-2} t} \int_{0}^{t} \sin ^{d-2} u\left(B_{t}(\mathfrak{D} g, \varpi)-\mathfrak{D} g(\varpi)\right) d u, \\
& \alpha(n)=\int_{0}^{\pi} M_{n ; j, i, s}(\theta) \sin ^{d-2} \theta d \theta \int_{0}^{\theta} \frac{d t}{\sin ^{d-2} t} \int_{0}^{t} \sin ^{d-2} u d u \\
& \quad \asymp \int_{0}^{\pi} M_{n ; j, i, s}(\theta) \sin ^{d-2} \theta d \theta \int_{0}^{\theta} \frac{t \sin ^{d-2} \xi}{\sin ^{d-2} t} d t \\
& \asymp \int_{0}^{\pi} \theta^{2} M_{n ; j, i, s}(\theta) \sin ^{d-2} \theta d \theta \asymp n^{-2} \quad(0<\xi<t) .
\end{aligned}
$$

Using Lemma 3.3, and the expression of $B_{t}(\mathfrak{D} g, \varpi)-\mathfrak{D} g$, we obtain

$$
\left\|\Psi_{\theta}(g)\right\|_{p_{m}} \leq C(d, j, i, s) \theta^{4}\left\|\mathfrak{D}^{2} g\right\|_{p_{m}} .
$$

By Lemma 3.2, and the Hölder-Minkowski inequality we get

$$
\begin{aligned}
& \left\|\int_{0}^{\pi} M_{n ; j, i, s}(\theta) \sin ^{d-2} \theta \Psi_{\theta}(g, \varpi) d \theta\right\|_{p_{m}} \\
& \quad \leq C(d, j, i, s)\left\|\mathfrak{D}^{2} g\right\|_{p_{m}} \int_{0}^{\pi} \theta^{4} M_{n ; j, i, s}(\theta) \sin ^{d-2} \theta d \theta \leq C(d, j, i, s) n^{-4}\left\|\mathfrak{D}^{2} g\right\|_{p_{m}} .
\end{aligned}
$$

Consequently, by (3.7), (3.8), and (3.9), we get

$$
\left\|J_{n ; j, i, s} g-g-\alpha(n) \mathfrak{D} g\right\|_{p_{m}} \leq C(d, j, i, s) n^{-4}\left\|\mathfrak{D}^{2} g\right\|_{p_{m}} .
$$

By Lemma 2.2, we have

$$
\begin{aligned}
& \left\|J_{n ; j, i, s} g-g-\alpha(n) \mathfrak{D} g\right\|_{B a} \\
& \quad \inf _{\alpha>0}\left\{\alpha: \sum_{m=1}^{\infty} \frac{a_{m}}{\alpha^{m}}\left\|J_{n ; j, i, s} g-g-\alpha(n) \mathfrak{D} g\right\|_{p_{m}}^{m} \leq 1\right\} \\
& \quad \leq \inf _{\alpha>0}\left\{\alpha: \sum_{m=1}^{\infty} \frac{a_{m}}{\alpha^{m}} C(d, j, i, s) n^{-4}\left\|\mathfrak{D}^{2} g\right\|_{p_{m}}^{m} \leq 1\right\} \\
& \quad \leq \inf _{\alpha>0}\left\{\alpha: \sum_{m=1}^{\infty} \frac{q^{m} \cdot C^{m}}{\alpha^{m}} C(d, j, i, s) n^{-4}\left\|\mathfrak{D}^{2} g\right\|_{B a}^{m} \leq 1\right\} \\
& \quad \leq C(d, j, i, s, q, \mu) n^{-4}\left\|\mathfrak{D}^{2} g\right\|_{B a} .
\end{aligned}
$$

The proof is completed. 


\section{Main results}

Theorem 4.1 Suppose that $f \in B a:=\bigcap_{m=1}^{\infty} L_{p_{m}}(\mathbb{S}), m=1,2, \ldots, 1 \leq p_{m} \leq \infty, J_{n ; j, i, s}(f ; \varpi)$ be the Jackson-Matsuoka polynomial on the unit sphere based on the Jackson-Matsuoka kernel, $4 i s>d+3,2 \lambda=d-2, j \geq i$. Then

$$
\left\|J_{n ; j, i, s}(f)-f\right\|_{B a} \leq C(d, j, i, s) \omega\left(f ; n^{-1}\right)_{B a} .
$$

Proof Since $\left(f_{0} * M_{n ; j, i, s}\right)(\varpi)=1$ for $f_{0}(\varpi)=1$, Therefore, we have

$$
\begin{aligned}
& \left\|J_{n ; j, i, s}(f)-f\right\|_{B a} \\
& \quad=\inf _{\alpha>0}\left\{\alpha: \sum_{m=1}^{\infty} \frac{a_{m}}{\alpha^{m}}\left\|J_{n ; j i, i,}(f)-f\right\|_{p_{m}}^{m} \leq 1\right\} \\
& \quad \leq \inf _{\alpha>0}\left\{\alpha: \sum_{m=1}^{\infty} \frac{a_{m}}{\alpha^{m}}\left\|\int_{0}^{\pi} M_{n ; j, i, s}(\theta)\left(f(x)-T_{\theta}(f ; x)\right) \sin ^{2 \lambda} \theta d \theta\right\|_{p_{m}}^{m} \leq 1\right\} \\
& \quad \leq \inf _{\alpha>0}\left\{\alpha: \sum_{m=1}^{\infty} \frac{a_{m}}{\alpha^{m}}\left(\int_{0}^{\pi}\left\|f-T_{\theta}(f)\right\|_{p_{m}} M_{n ; j, i, s}(\theta) \sin ^{2 \lambda} \theta d \theta\right)^{m} \leq 1\right\} \\
& \quad \leq \inf _{\alpha>0}\left\{\alpha: \sum_{m=1}^{\infty} \frac{q^{m} \cdot C^{m}}{\alpha^{m}}\left(\int_{0}^{\pi}\left\|f-T_{\theta}(f)\right\|_{B a} M_{n ; j, i, s}(\theta) \sin ^{2 \lambda} \theta d \theta\right)^{m} \leq 1\right\} .
\end{aligned}
$$

Splitting the integral on $[0, \pi]$ into two integrals on $[0,1 / n]$ and $[1 / n, \pi]$, respectively, and using the definition of $\omega(f ; t)_{B a}$, we conclude that

$$
\left\|f-T_{\theta}(f)\right\|_{B a} \leq \omega\left(f ; n^{-1}\right)_{B a}+\int_{1 / n}^{\pi} \omega(f ; \theta)_{B a} M_{n ; j, i, i}(\theta) \sin ^{2 \lambda} \theta d \theta .
$$

From Corollary 2.4 we have, for $\theta \geq n^{-1}$,

$$
\omega(f ; \theta)_{B a}=\omega\left(f ; n^{-1}\right)_{B a} \leq C \max \left\{1, n^{2} \theta^{2}\right\} \omega\left(f ; n^{-1}\right)_{B a} \leq C n^{2} \theta^{2} \omega\left(f ; n^{-1}\right)_{B a} .
$$

By (4.3), (4.4), and Lemma 3.1, we get

$$
\left\|f-T_{\theta}(f)\right\|_{B a} \leq C \omega(f ; \theta)_{B a} .
$$

Therefore, by (4.2), (4.5), we have

$$
\begin{aligned}
& \left\|J_{n ; j, i, s}(f)-f\right\|_{B a} \\
& \quad \leq \inf _{\alpha>0}\left\{\alpha: \sum_{m=1}^{\infty} \frac{q^{m} \cdot C^{m}}{\alpha^{m}}\left(\int_{0}^{\pi} \omega\left(f ; n^{-1}\right)_{B a} M_{n ; j, i, s}(\theta) \sin ^{2 \lambda} \theta d \theta\right)^{m} \leq 1\right\} \\
& \quad=\inf _{\alpha>0}\left\{\alpha: \sum_{m=1}^{\infty} \frac{q^{m} \cdot C^{m}}{\alpha^{m}}\left(\omega\left(f ; n^{-1}\right)_{B a}\right)^{m} \leq 1\right\} \\
& \leq C(d, j, i, s, q, \mu) \omega\left(f ; n^{-1}\right)_{B a} .
\end{aligned}
$$


Theorem 4.2 Suppose that $f \in B a:=\bigcap_{m=1}^{\infty} L_{p_{m}}(\mathbb{S}), 1 \leq p_{m} \leq \infty, J_{n ; j, i, s}(f ; x)$ is the JacksonMatsuoka polynomial on the unit sphere based on the Jackson-Matsuoka kernel, 4 is $>d+3$, $2 \lambda=d-2, j \geq i, 0<\alpha<1$. Then the following statements are equivalent:

$$
\begin{aligned}
& \text { (1) }\left\|J_{n ; j, i, s}(f)-f\right\|_{B a}=O\left(n^{-\alpha}\right), \quad n \geq 2 ; \\
& \text { (2) } \omega\left(f ; n^{-1}\right)_{B a}=O\left(t^{\alpha}\right), \quad 0<t<1 .
\end{aligned}
$$

Proof By Theorem 4.1, we have (2) $\Rightarrow(1)$. Now, we prove (1) $\Rightarrow$ (2). Let $r$ be a fixed positive integer, defined by

$$
J_{n ; j, i, s}^{r}(f ; \varpi):=\sum_{k=0}^{r}\left(\int_{0}^{\pi} M_{n ; j, i, s}(\theta) Q_{k}^{\lambda}(\cos \theta) \sin ^{2 \lambda} \theta d \theta\right)^{r} Y_{k}(f ; \varpi)
$$

By orthogonality of the orthogonal projector $Y_{k}$, we have

$$
\begin{aligned}
J^{r+l}(f)= & \sum_{k=0}^{r}\left(\int_{0}^{\pi} M_{n ; j, i, s}(\theta) Q_{k}^{\lambda}(\cos \theta) \sin ^{2 \lambda} \theta d \theta\right)^{r} \\
& \times Y_{k}\left(\sum_{v=0}^{r}\left(\int_{0}^{\pi} M_{n ; j, i, s}(\theta) Q_{\nu}^{\lambda}(\cos \theta) \sin ^{2 \lambda} \theta d \theta\right)^{l} Y_{v}(f)\right) \\
= & J_{n ; j, i, s}^{r}\left(J_{n ; j, i, s}^{l}(f)\right) .
\end{aligned}
$$

Let $g=J_{n ; j, i, s}^{r}(f)$, by (4.9) we get

$$
\begin{aligned}
\|f-g\|_{B a} & =\inf _{\alpha>0}\left\{\alpha: \sum_{m=1}^{\infty} \frac{a_{m}}{\alpha^{m}}\|f-g\|_{p_{m}}^{m} \leq 1\right\} \\
& =\inf _{\alpha>0}\left\{\alpha: \sum_{m=1}^{\infty} \frac{a_{m}}{\alpha^{m}}\left\|f-J_{n ; j, i, s}^{r}(f)\right\|_{p_{m}}^{m} \leq 1\right\} \\
& \leq \inf _{\alpha>0}\left\{\alpha: \sum_{m=1}^{\infty} \frac{a_{m}}{\alpha^{m}}\left(\sum_{k=1}^{r}\left\|J_{n ; j, i, s}^{k-1}(f)-J_{n ; j, i, s}^{k}(f)\right\|_{p_{m}}\right)^{m} \leq 1\right\} \\
& \leq \inf _{\alpha>0}\left\{\alpha: \sum_{m=1}^{\infty} \frac{a_{m}}{\alpha^{m}}\left(C(d, j, i, s) \sum_{k=1}^{r}\left\|J_{n ; j, i, s}^{k-1}\left(f-J_{n ; j, i, s}(f)\right)\right\|_{p_{m}}\right)^{m} \leq 1\right\} \\
& \leq \inf _{\alpha>0}\left\{\alpha: \sum_{m=1}^{\infty} \frac{a_{m}}{\alpha^{m}} C(d, j, i, s) r\left\|f-J_{n ; j, i, s}(f)\right\|_{p_{m}}^{m} \leq 1\right\} \\
& \leq \inf _{\alpha>0}\left\{\alpha: \sum_{m=1}^{\infty} \frac{q^{m} \cdot C_{1}^{m}(d, j, i, s, r)}{\alpha^{m}}\left\|f-J_{n ; j, i, s}(f)\right\|_{B a}^{m} \leq 1\right\} \\
& \leq C(d, j, i, s, r, q, \mu)\left\|f-J_{n ; j i, i}(f)\right\|_{B a},
\end{aligned}
$$

where $J_{n ; j, i, s}^{0}(f)=f$.

On the other hand,

$$
\left\|\mathfrak{D} J_{n ; j, i, s}^{r}(f)\right\|_{p_{m}} \leq \sum_{k=0}^{r} k(k+d-2)\left(\int_{0}^{\pi} M_{n ; j, i, s}(\theta)\left|Q_{k}^{\lambda}(\cos \theta)\right| \sin ^{2 \lambda} \theta d \theta\right)^{r} Y_{k}(f) .
$$


Note that $[10]$

$$
\left|Q_{k}^{\lambda}(\cos \theta)\right| \equiv\left|\frac{C_{k}^{\lambda}(\cos \theta)}{C_{k}^{\lambda}(1)}\right| \leq C \min \left\{(k \theta)^{-1}, 1\right\}
$$

For $k \theta \geq 1$, from (2.4) we have

$$
\begin{aligned}
& \left\|\mathfrak{D J}_{n ; j, i, s}^{r}(f)\right\|_{p_{m}} \\
& \quad \leq C(d, j, i, s)\left\|\sum_{k=0}^{r} k(k+d-2) k^{-r \lambda}\left(\int_{0}^{\pi} M_{n ; j ;, j, s}(\theta) \theta^{-\lambda} \sin ^{2 \lambda} \theta d \theta\right)^{r} Y_{k}(f)\right\|_{p_{m}} \\
& \quad \leq C(d, j, i, s) n^{r \lambda}\|f\|_{p_{m}} \sum_{k=0}^{\infty} k^{2-r \lambda} \leq C(d, j, i, s) n^{r \lambda}\|f\|_{p_{m}}
\end{aligned}
$$

holds for $r>\frac{6}{d-2}$. For $k \theta<1$, by Lemma 3.2, we get

$$
\begin{aligned}
& \left\|\mathcal{D}_{n ; j, i, s}^{r}(f)\right\|_{p_{m}} \\
& \quad \leq\left\|\sum_{k=0}^{r}\left(\int_{0}^{\pi} M_{n ; j, i, s}(\theta) \theta^{-\frac{2}{r}}\left(\theta^{2} k(k+d-2)\right)^{\frac{1}{r}}\left|Q_{k}^{\lambda}(\cos \theta)\right| \sin ^{2 \lambda} \theta d \theta\right)^{r} Y_{k}(f)\right\|_{p_{m}} \\
& \leq C(d, j, i, s)\left\|\sum_{k=0}^{r}\left(\int_{0}^{\pi} M_{n ; j, i, s}(\theta) \theta^{-\frac{2}{r}}\left((k \theta)^{2}\right)^{\frac{2}{r}} \sin ^{2 \lambda} \theta d \theta\right)^{r} Y_{k}(f)\right\|_{p_{m}} \\
& \quad \leq C(d, j, i, s)\left\|\sum_{k=0}^{r}\left(\int_{0}^{\pi} M_{n ; j, i, s}(\theta) \theta^{-\frac{2}{r}} \sin ^{2 \lambda} \theta d \theta\right)^{r} Y_{k}(f)\right\|_{p_{m}} \\
& \quad \leq C(d, j, i, s) n^{2}\left\|\sum_{k=0}^{\infty} Y_{k}(f)\right\|_{p_{m}} \leq C(d, j, i, s) n^{2}\|f\|_{p_{m}} .
\end{aligned}
$$

Consequently, the inequality

$$
\left\|\mathfrak{D} J_{n ; j, i, s}^{r}(f)\right\|_{p_{m}} \leq C(d, j, i, s) n^{2}\|f\|_{p_{m}}
$$

holds uniformly for $r>\frac{6}{d-2}$. Thereby

$$
\begin{aligned}
& \left\|\mathcal{D J}_{n ; j, i, s}^{r}(f)\right\|_{B a} \\
& \quad=\inf _{\alpha>0}\left\{\alpha: \sum_{m=1}^{\infty} \frac{a_{m}}{\alpha^{m}}\left\|\mathfrak{D} J_{n ; j, i, s}^{r}(f)\right\|_{p_{m}} \leq 1\right\} \\
& \quad \leq \inf _{\alpha>0}\left\{\alpha: \sum_{m=1}^{\infty} \frac{a_{m}}{\alpha^{m}} C(d, j, i, s) n^{2}\|f\|_{p_{m}} \leq 1\right\} \\
& \quad \leq \inf _{\alpha>0}\left\{\alpha: \sum_{m=1}^{\infty} \frac{q^{m} \cdot C^{m}}{\alpha^{m}} C(d, j, i, s) n^{2}\|f\|_{B a} \leq 1\right\} \\
& \quad \leq C(d, j, i, s, q, \mu) n^{2}\|f\|_{B a} .
\end{aligned}
$$


Without loss of generality, we may assume $r_{1}>\frac{6}{d-2}, r>r_{1}+\frac{6}{d-2}$. Using Lemma 3.4 and (4.9), we have

$$
\begin{aligned}
& \alpha(n)\left\|\mathfrak{D} J_{n ; j, i, s}^{r}(f)\right\|_{B a}=\left\|\alpha(n) \mathfrak{D} J_{n ; j ;, i, s}^{r}(f)\right\|_{B a} \\
& \leq\left\|J_{n ; j, i, s}^{r}(f)-f\right\|_{B a}+C(d, j, i, s) n^{-2}\left\|\mathfrak{D}^{2} J_{n ; j, i, s}^{r}(f)\right\|_{B a} \\
& \leq r\left\|J_{n ; j, i, s}(f)-f\right\|_{B a}+C(d, j, i, s) n^{-2}\left\|\mathfrak{D}^{2} J_{n ; j, i, s}^{r-r_{1}}(f)\right\|_{B a} \\
& \leq r\left\|J_{n ; j, i, s}(f)-f\right\|_{B a} \\
& +C(d, j, i, s)\left(n^{-2}\left\|\mathfrak{D} J_{n ; j, i, s}^{r}(f)\right\|_{B a}+n^{-2}\left\|J_{n ; j, i, s}^{r}(f)-J_{n ; j, i, s}^{r-r_{1}}(f)\right\|_{B a}\right) \\
& \leq r\left\|J_{n ; j, i, s}(f)-f\right\|_{B a} \\
& \left.+C(d, j, i, s)\left(n^{-2} \| \mathfrak{D}\right)_{n ; j, i, s}^{r}(f)\left\|_{B a}+\right\| J_{n ; j ; i, s}^{r_{1}}(f)-f \|_{B a}\right) \\
& \leq C(d, j, i, s, r)\left(\left\|J_{n ; j, i, s}(f)-f\right\|_{B a}+n^{-2}\left\|\mathfrak{D} J_{n ; j, i, s}^{r}(f)\right\|_{B a}\right) \\
& \leq C(d, j, i, s, r, q, \mu)\left(\left\|J_{n ; j ;, i, s}(f)-f\right\|_{B a}+\|f\|_{B a}\right) .
\end{aligned}
$$

Consequently, considering $n^{-2}\left\|\mathfrak{D} J_{n ; j, i, s}^{r}(f)\right\|_{B a} \leq C(d, j, i, s, r, q, \mu)\left\|f-J_{n ; j, i, s}(f)\right\|_{B a}$, by the definition of $K\left(f ; t^{2}\right)_{B a}$, and Theorem 2.3, we have

$$
\begin{aligned}
\omega\left(f ; n^{-1}\right)_{B a} & \leq C K\left(f ; n^{-2}\right)_{B a} \\
& \leq C\left(\left\|f-J_{n ; j, i, s}^{r}(f)\right\|_{B a}+n^{-2}\left\|\mathfrak{D} J_{n ; j, i, s}^{r}(f)\right\|_{B a}\right) \\
& \leq C(d, j, i, s, r, q, \mu)\left\|f-J_{n ; j, i, s}(f)\right\|_{B a} .
\end{aligned}
$$

In view of (4.7), we get

$$
\omega\left(f ; n^{-1}\right)_{B a} \leq C(d, j, i, s, r, q, \mu) n^{-\alpha} .
$$

Let $(n+1)^{-1}<t \leq n^{-1}$, we have

$$
\begin{aligned}
\omega(f ; t)_{B a} & \leq \omega\left(f ; n^{-1}\right)_{B a} \leq C(d, j, i, s, r, q, \mu)\left(\frac{n}{n+1}\right)^{-\alpha}(n+1)^{-\alpha} \\
& \leq C(d, j, i, s)(n+1)^{-\alpha} \leq C(d, j, i, s, r, q, \mu) t^{\alpha} .
\end{aligned}
$$

The proof is completed.

\section{Competing interests}

The authors declare that they have no competing interests.

Authors' contributions

All authors contributed equally to the writing of this paper. All authors read and approved the final manuscript.

\section{Author details}

'Department of Mathematics, Taizhou University, Taizhou, Zhejiang 317000, China. ${ }^{2}$ School of Science, China University of Mining and Technology, Beijing, 100083, China. 


\section{Acknowledgements}

The authors would like to thank the anonymous referees for their valuable comments, remarks, and suggestions, which greatly helped us to improve the presentation of this paper and made it more readable. Project was supported by the Natural Science Foundation of China (Grant No. 10671019), the Zhejiang Provincial Natural Science Foundation (Grant No. LY12A01008), and the Cultivation Fund of Taizhou University.

Received: 26 April 2014 Accepted: 10 October 2014 Published: 21 October 2014

\section{References}

1. Ding, XX, Luo, PZ: Ba spaces and some estimates of Laplace operator. J. Syst. Sci. Math. Sci. 1, 9-33 (1981)

2. Freedden, W, Gervens, T, Schreiner, M: Constructive Approximation on the Sphere. Oxford University Press, New York (1998)

3. Wang, KY, Li, LQ: Harmonic Analysis and Approximation on the Unit Sphere. Science Press, Beijing (2000)

4. Müller, C: Spherical Harmonics. Lecture Notes in Mathematics, vol. 17. Springer, Berlin (1971)

5. Berens, H, Butzer, PL, Pawelke, S: Limitierungsverfahren von Reihen mehrdimensionaler Kugelfunktionen und deren Saturationsverhalten. Publ. Res. Inst. Math. Sci., Ser. A 4, 201-268 (1968)

6. Pawelke, S: Über die Approximationsordnung bei Kugelfunktionen und algebraischen Polynomen. Tohoku Math. J. 24, 473-486 (1972)

7. Matsuoka, Y: On the approximation of functions by some singular integrals. Tohoku Math. J. 18, 13-43 (1966)

8. Chen, WZ: Approximation Theory of Operators. Xiamen University Publishing House, Xiamen (1989) (in Chinese)

9. Ditzian, Z, Runovskii, K: Averages on caps of $\mathbb{S}^{d-1}$. J. Math. Anal. Appl. 248, 260-274 (2000)

10. Belinsky, E, Dai, F, Ditzian, Z: Multivariate approximating averages. J. Approx. Theory 125, 85-105 (2003)

doi:10.1186/1029-242X-2014-419

Cite this article as: Feng and Feng: Direct and converse results in the Ba space for Jackson-Matsuoka polynomials on the unit sphere. Journal of Inequalities and Applications 2014 2014:419.

\section{Submit your manuscript to a SpringerOpen ${ }^{\circ}$ journal and benefit from:}

- Convenient online submission

- Rigorous peer review

Immediate publication on acceptance

Open access: articles freely available online

- High visibility within the field

- Retaining the copyright to your article 